\title{
Variability in the Propagation Phase of CFD-Based Noise Prediction: Summary of Results from Category 8 of the BANC-III Workshop
}

\author{
Leonard Lopes*, Stéphane Redonnet $†$ Taro Imamura \\ Tomoaki Ikedå Nikolas Zawodny, Guilherme Cunha\|
}

\begin{abstract}
The usage of Computational Fluid Dynamics (CFD) in noise prediction typically has been a two part process: accurately predicting the flow conditions in the near-field and then propagating the noise from the near-field to the observer. Due to the increase in computing power and the cost benefit when weighed against wind tunnel testing, the usage of CFD to estimate the local flow field of complex geometrical structures has become more routine. Recently, the Benchmark problems in Airframe Noise Computation (BANC) workshops have provided a community focus on accurately simulating the local flow field near the body with various CFD approaches. However, to date, little effort has been given into assessing the impact of the propagation phase of noise prediction. This paper includes results from the BANC-III workshop which explores variability in the propagation phase of CFD-based noise prediction. This includes two test cases: an analytical solution of a quadrupole source near a sphere and a computational solution around a nose landing gear. Agreement between three codes was very good for the analytic test case, but CFD-based noise predictions indicate that the propagation phase can introduce $3 \mathrm{~dB}$ or more of variability in noise predictions.
\end{abstract}

\section{Nomenclature}

English:

c speed of sound

$f \quad$ surface of a body $(f=0)$

$F_{i} \quad$ compact line dipole source term

$G \quad$ Green's function

$H(x) \quad$ Heaviside function

$L_{i} \quad$ surface dipole source term

$M \quad$ Mach number

$n_{i} \quad$ outward directed unit normal vector

$p^{\prime} \quad$ acoustic pressure

$p_{i j} \quad$ compressive stress tensor

$Q \quad$ monopole source term

$r \quad$ radiation vector

$S \quad$ integration surface

$t \quad$ observer time

$T_{i j} \quad$ quadrupole source term

$u_{i}, v_{i} \quad$ flow and surface velocity

\section{Greek:}

$\delta(x) \quad$ Dirac delta function

$\delta_{i j} \quad$ Kronecker delta

$\rho \quad$ fluid density

$\sigma \quad$ standard deviation

$\tau \quad$ retarded time

Subscript:

I incident field

$n \quad$ unit normal direction

$S \quad$ scattered field

$\infty \quad$ freestream quantity

Superscript:

' perturbation quantity

- generalized derivative

Symbol:

$\square^{2} \quad$ wave operator, $1 / c_{\infty}^{2} \partial^{2} / \partial t^{2}-\partial^{2} / \partial x_{i} \partial x_{i}$

\footnotetext{
*Research Aerospace Engineer, NASA Langley, leonard.v.lopes@nasa.gov, AIAA Member

${ }^{\dagger}$ Senior Scientist / Research Engineer, ONERA, stephane.redonnet@onera.fr, AIAA Member

$¥$ Associate Professor, The University of Tokyo, timat@mail.ecc.u-tokyo.ac.jp, AIAA Senior Member

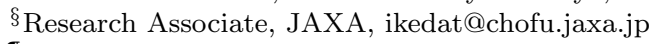

ๆ Research Aerospace Engineer, NASA Langley, nikolas.s.zawodny@nasa.gov, AIAA Member

"ISenior Scientist / Research Engineer, ONERA, guilherme.cunha@onera.fr
} 


\section{Introduction}

The study of noise from aircraft has historically progressed on three fronts: analytical theory, wind tunnel or field measurements, and computer-based applications including Computational Fluid Dynamics (CFD). Typically, utilizing CFD to predict noise involves a large calculation that captures the near-field fluctuations including acoustics and a post-processing algorithm that propagates the acoustics from the near-field to observer positions. Separating the computation into two parts allows the propagation phase of noise prediction to use reduced order models in order to save time and computer resources. However, there are several different approaches to noise propagation, each with benefits and assumptions that affect the overall result. This leads to uncertainty and variability that can be directly associated with the propagation phase of noise prediction.

The propagation of noise from a sound generating region to an observer position involves many problem areas such as scattering obstacles and medium heterogeneities such as shear layers. There are several different approaches to the propagation phase of noise prediction including Integral Methods (such as Kirchhoff extrapolations $^{1,2}$ and Acoustic Analogies ${ }^{3,4}$ ), Ray Methods (RM), ${ }^{5}$ Boundary Element Methods (BEM) ${ }^{6,7}$ Equivalent Source Methods (ESM) ${ }^{8,9}$ and Computational AeroAcoustics (CAA). ${ }^{10}$ While there has been effort in each area to categorize and validate approaches, there has not been a concentrated effort to compare the range of applicability and trade-offs across the methods.

Recently, the Benchmark problems for Airframe Noise Computations (BANC) workshop has focused on the validation of CFD-based approaches for noise prediction of airframe sources. ${ }^{11}$ The BANC workshop includes eight categories: the first seven focus on near-field computations of the flow field around airframe structures, such as the landing gear, flap, and trailing edges; Category 8 focuses on assessing the variability in the propagation phase of CFD-based noise prediction. This paper presents results from BANC-III Category 8. This includes applying different propagation approaches to two subcategories: the scattering of a quadrupole source by a sphere and the propagation of noise from a simplified nose landing gear. A summary of the BANCIII Category 8 participant's propagation methods is included, after which the result from each subcategory is shown. Finally, a Conclusion and Future Work section is presented that includes proposals for additional subcategories for follow-on efforts.

\section{Propagation Methods}

The noise propagation portion of CFD-based noise prediction involves using CFD to predict the timedependent near-field hydrodynamics and acoustics and a propagation algorithm to calculate a pressure time history at an observer position. Outside the near-field where the noise is generated, there can exist significant influences on the noise propagation, such as shielding/scattering bodies or flow heterogeneities. These reside in a mid-field region. Once outside the mid-field region, noise typically propagates through a homogeneous medium to an observer. Figure 1 shows a comparison between a simple propagation problem and a more realistic one. Figure 1a shows propagation with no body interactions and an immediate transition from near- to far-field. Figure 1b shows propagation with potential shielding and scattering interactions, as well as heterogeneous propagation effects in the mid-field. There are several different approaches to calculating the noise propagation, summarized in Figure 2. Each of the approaches must be provided a solution to the near-field region, including hydrodynamics and acoustics, and can provide a prediction at an observer position in the near- or far-field. Once a pressure time history at the observer is determined, post processing algorithms are applied so that a direct comparison to wind tunnel measurements can be performed. Due to participation in Category 8 of the BANC-III workshop, for brevity, only three approaches are included in this study: Integral Methods, Boundary Element Methods, and Computation AeroAcoustics. Following is a brief explanation of each. 


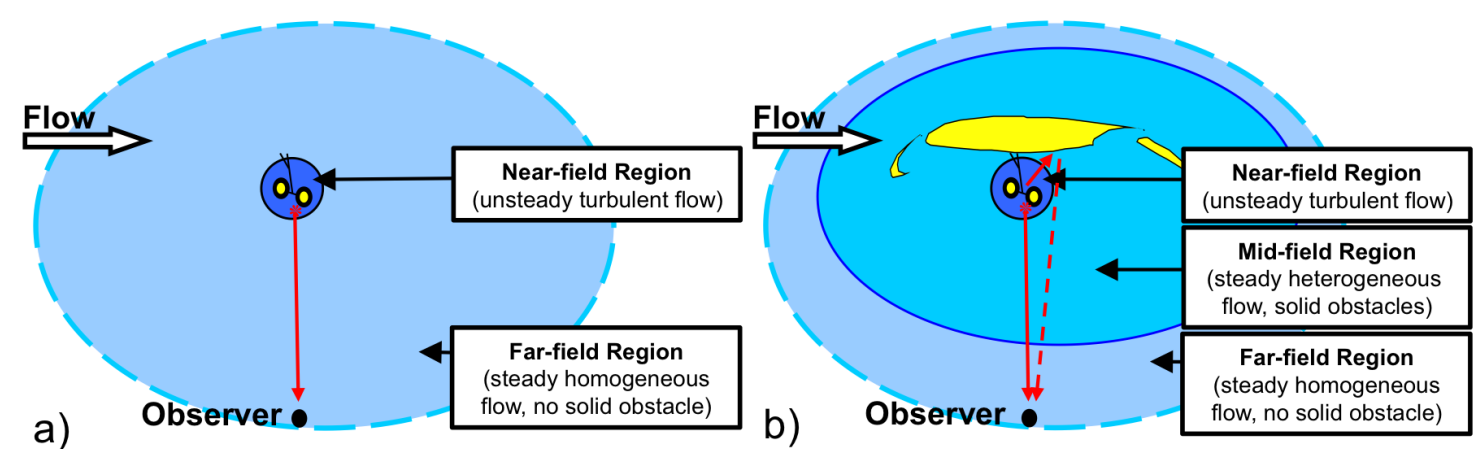

Figure 1. Summary of regions of CFD-based noise prediction. a) Noise generating region placed in isolation where no bodies influence noise propagation. b) Body exists near noise generating region influencing the noise propagation.

\section{A. Integral Methods (IM)}

Over the past 30 years, Integral Methods (IMs) have provided a relatively computationally inexpensive method for propagating noise from a nearfield region to an observer location. Historically, the most popular IMs are based on a reformulation of the Navier-Stokes equation using Generalized Functions, ${ }^{12}$ called the Ffowcs Williams and Hawkings (FW-H) equation. The FW-H equation utilizes Lighthill's acoustic analogy to formulate an inhomogenous wave equation where source terms are defined as discontinuous surfaces and a volume region external to the surface. The FW-H equation, Eq. 1, includes two surface source terms and one volume source term. The surface source terms are identified by the delta function, $\delta(x)$, and the volume source term is identified by the Heaviside function, $H(x)$.

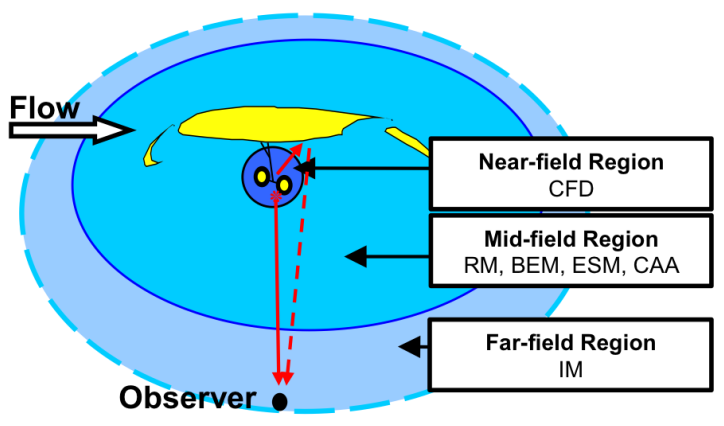

Figure 2. Summary of coupled CFD and propagation components of CFD-based noise prediction. The source terms on the right hand side of the FW-

$\mathrm{H}$ equation often are called the monopole, dipole, and quadrupole terms respectively. The surface $f$, shown in Fig. 3, can be impermeable, such as a rotor blade surface, or permeable, such as a computational surface surrounding the entire rotor which provides flowfield information. The general source terms are shown in Eq. 2, and the simplified source terms valid for impermeable surfaces are shown in Eq 3.

$$
\begin{gathered}
\bar{\square}^{2} p^{\prime}=\frac{\partial}{\partial t}\{Q \delta(f)\}-\frac{\partial}{\partial x_{i}}\left\{L_{i} \delta(f)\right\}+\frac{\bar{\partial}^{2}}{\partial x_{i} \partial x_{j}}\left\{T_{i j} H(f)\right\} \\
Q=\rho_{\infty} v_{n}+\rho\left(u_{n}-v_{n}\right), \quad L_{i}=p_{i j} n_{j}+\rho u_{i}\left(u_{n}-v_{n}\right), \quad T_{i j}=\rho u_{i} u_{j}+p_{i j}-c_{\infty}^{2} \rho^{\prime} \delta_{i j} \\
Q=\rho_{\infty} v_{n}, \quad L_{i}=p_{i j} n_{j}, \quad T_{i j}=\rho u_{i} u_{j}+p_{i j}-c_{\infty}^{2} \rho^{\prime} \delta_{i j}
\end{gathered}
$$

Integral methods often ignore the quadrupole term with the assumption that all noise sources and nonuniform flow propagation effects are included within and thereby captured by the surface terms. IMs also often apply a free-space Green's function by assuming a uniform propagation region; therfore, IMs cannot account for the influence of bodies or medium heterogeneities. These assumptions often lead to inaccuracies in the prediction. However, usage of integral methods have provided a very reliable prediction of the propagation with a relatively small amount of computational effort. 


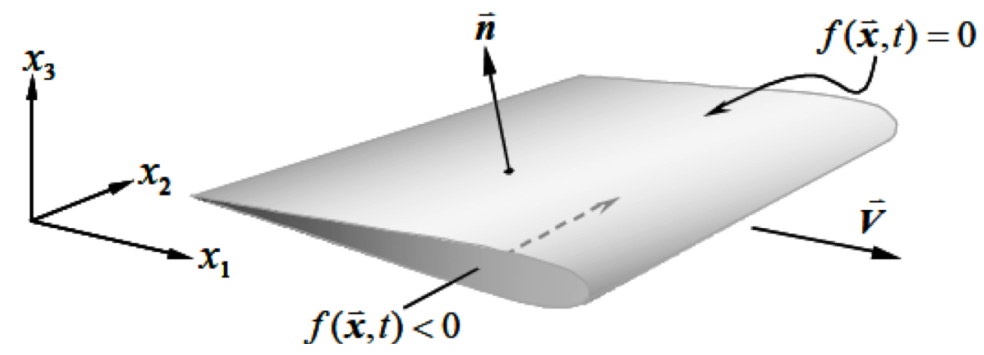

Figure 3. Description of a surface in motion by $f(\mathbf{x}, t)=0, \bar{n}=\frac{\partial f}{\partial \mathbf{x}}, f(\mathbf{x}, t)<0$ inside the surface, and $f(\mathbf{x}, t)>0$ outside the surface.

\section{B. Boundary Element Methods (BEM)}

Boundary Element Methods (BEMs) discretize the surface of any shielding/scattering body and combine the convective wave equation, shown in Eq. 4, with a Green's function, shown in Eq. 5. A useful equation for the pressure can be obtained by subtracting the pressure times Eq. 5 from the Green's function multiplied by Eq. 4. If the pressure is assumed to be comprised of an incident field and a scattered field, then Eqs. 6, 7, and 8 provide integral relations for the two components of the pressure. ${ }^{7}$ The incident field is calculated similar to an IM, by assuming a free-space Green's function. The scattered field is calculated by integrating surface elements along the scattering body, where each element contributes to the scattered field accounting for its own incident field from all other elements. In this way, BEMs can account for a shielding/scattering body; however, BEMs have yet to include effects such as flow heterogeneities. Similar to ESMs, BEMs have had difficulty at higher frequencies due to a need for reduced element and time step size, but as computer power increases BEMs have gained significant traction.

$$
\begin{gathered}
\left(\frac{\partial}{\partial t}+u_{i} \cdot \frac{\partial}{\partial x_{i}}\right)^{2} p^{\prime}-c^{2} \frac{\partial^{2} p^{\prime}}{\partial x_{i}^{2}}=q\left(y_{i}, t\right) \\
\left(\frac{\partial}{\partial t}+u_{i} \cdot \frac{\partial}{\partial x_{i}}\right)^{2} G-c^{2} \frac{\partial^{2} G}{\partial x_{i}^{2}}=\delta\left(x_{i}-y_{i}\right) \delta\left(t-\tau+\left|x_{i}-y_{i}\right| / c\right) \\
p^{\prime}\left(x_{i}, t\right)=p_{I}^{\prime}\left(x_{i}, t\right)+p_{S}^{\prime}\left(x_{i}, t\right) \\
p_{I}^{\prime}\left(x_{i}, t\right)=\int_{-\infty}^{t} \int_{V} G q\left(y_{i}, \tau\right) d y_{i} d \tau \\
p_{S}^{\prime}\left(x_{i}, t\right)=c^{2} \int_{-\infty}^{t} \int_{S}\left[G \frac{\partial p^{\prime}}{\partial n}-p^{\prime} \frac{\partial G}{\partial n}\right] d S d \tau- \\
\int_{-\infty}^{t} \int_{S}\left[G\left(\frac{\partial p^{\prime}}{\partial \tau}+u_{i} \cdot \frac{\partial p^{\prime}}{\partial y_{i}}\right)-p^{\prime}\left(\frac{\partial G}{\partial \tau}+u_{i} \cdot \frac{\partial G}{\partial y_{i}}\right)\right] u_{n} d S d \tau
\end{gathered}
$$

\section{Computational AeroAcoustics (CAA)}

Computational AeroAcoustic (CAA) methods rely on a discretized form of the linearized Euler equations to account for both acoustic reflection/diffraction by impermeable obstacles and refraction by flow heterogeneities. This is in contrast to other techniques that can only model obstacles (such as the BEM or ESM), or far-field propagation (such as an IM). A typical situation where an acoustic hybrid approach can be advantageously applied is external noise problems where the propagation phase occurs within a complex environment, such as the airframe noise emission by aircraft appendages (e.g. landing gear, etc.). Indeed, once their generation has been properly simulated, acoustic waves may then be transferred to a CAA solver, for the latter to propagate them through the mid-field, while accounting for all the installation effects induced by either the airframe elements (e.g. reflection/diffraction) or the airflow that surrounds the latter (e.g. convection/refraction). 
CAA methods usually rely on an arbitrary splitting of the usual physical quantities (primitive variables) into both a perturbed (subscript $p$ ) and a mean (or background, subscript $o$ ) component.

$$
\mathbf{q}=\mathbf{q}_{o}+\mathbf{q}_{p}
$$

where each q quantity is composed of its associated density, $\rho$, velocity, $\mathbf{v}$, and pressure, $p$. Invoking the classical small perturbation hypothesis, the background field, $\mathrm{q}_{o}=\left(\rho_{o}, \mathbf{v}_{o}, \mathbf{p}_{o}\right)$, can be considered as insensitive to the evolution of its perturbed counterpart, $\mathrm{q}_{p}=\left(\rho_{p}, \mathbf{v}_{p}, \mathbf{p}_{p}\right)$, as long as the latter remains sufficiently small in terms of amplitude. This allows the derivation of an equation governing solely the perturbed field, $\mathrm{q}_{p}$, whilst accounting for its background counterpart, $\mathrm{q}_{o}$ :

$$
\partial_{t} \mathbf{u}_{p}\left(\mathbf{q}_{o}, \mathbf{q}_{p}\right)+\nabla \mathbf{F}_{p}\left(\mathbf{q}_{o}, \mathbf{q}_{p}\right)=0
$$

In the above equations, $\mathbf{u}_{p}$ stands for the perturbed flow-field vector, and $\mathbf{F}_{p}$ stands for its associated convective flux matrices. For conciseness, these terms are not detailed here but can be found in Ref. 10. Equation 10 is then discretized in space and time and solved numerically; the $\mathbf{u}_{p}$ term being advanced in time while accounting for the mean flow and other effects (boundary conditions, etc). Note that the space/time discretization is usually handled with the help of high-order schemes, optimized for minimal dissipation and dispersion of the numerically propagated acoustic waves.

\section{BANC-III Category 8}

The goal of Category 8 of the BANC workshop is to quantify variability in the propagation phase of CFD-based noise prediction; this includes resources required, computation time, and accuracy of the result. Toward that end, two subcategories were created to quantify the variability. Subcategory 1, Scattering of Noise by a Sphere, was created as a simple test case to quantify the execution time, resources, and accuracy of each approach compared to an analytical solution. Subcategory 2, Nose Landing Gear, was created to assess the variability in the results when provided a common CFD solution of a complex noise generating region.

\section{Participants}

There were four participants in the BANC-III Category 8 effort: JAXA's upacsAcoustics IM solver, ${ }^{13}$ NASA's ANOPP2-F1A IM solver, ${ }^{14}$ ODU's TD-FAST BEM solver, ${ }^{15}$ and ONERA's sAbrinA CAA solver. ${ }^{7}$ Following is a brief description of each code.

\section{1. upacsAcoustics}

The JAXA's upacsAcoustics code is an Integral Method solver that utilizes a convective form of Farassat's Formulation 1A to solve for the acoustic pressure in double precision at an observer location provided the local flow conditions around a body in uniform, rectilinear flight. Only the monopole and dipole terms are included. The quadrupole term is ignored with the assumption that the freestream Mach number is low. The code uses a second-order spatial and temporal central differencing scheme with a first-order retarded time calculation.

\section{ANOPP2-F1A}

ANOPP2-F1A is the Formulation 1A solver incorporated in the Aircraft NOise Prediction Program 2 (ANOPP2). ${ }^{14}$ ANOPP2 is a mixed-fidelity acoustic framework, capable of combining high-resolution methods, such as those based on CFD computations, and low-resolution empirical methods, such as those based on experimental data and historical methods, for the application of acoustic research and Multi-disciplinary Design Analysis and Optimization (MDAO). ${ }^{16}$ ANOPP2-F1A utilizes a second-order central differencing scheme for spatial and temporal derivatives and a first-order retarded time calculation, both in single precision. ANOPP2-F1A is capable of predicting the near- and far-field acoustic pressure at an observer location provided the appropriate flow data on an input acoustic data surface which may be undergoing complex motions. The acoustic data surface may be node- or cell-centered, rigid or deforming, ${ }^{17}$ structured or unstructured and may also be undergoing complex motions such as those found in rotorcraft applications. In addition to Formulation 1A, ANOPP2 also includes Formulations $1,{ }^{18} 2 \mathrm{~B},{ }^{19} \mathrm{G} 1, \mathrm{G} 1 \mathrm{~A},{ }^{20}$ and V1A. ${ }^{21}$ 


\section{TD-FAST}

TD-FAST (Time-Domain Fast Acoustic Scattering Toolkit) is a time domain boundary integral equation solver. ${ }^{7}{ }^{15}$ It has the distinct advantage that acoustic scattering and shielding solutions at all frequencies within the numerical resolution can be captured by one single time domain computation in double precision. In addition, broadband sources and time dependent transient signals can be simulated and studied directly in TD-FAST. It also can be used to couple with nonlinear CFD simulations where many frequencies are generated. Solution of the boundary integral equation in TD-FAST requires only unstructured surface triangular and quadrilateral elements. Computational complexity in TD-FAST is reduced by the Time Domain Propagation and Distribution (TDPD) algorithm that has been developed based on the delay- and amplitude-compensated acoustic field. Computation in TD-FAST is further accelerated by a use of general purpose GPU computing. TD-FAST has been implemented in a multi-CPU and multi-GPU platform.

\section{4. $\operatorname{sAbrin} A$}

sAbrin ${ }^{10,22-26}$ is a structured, time-accurate CAA code that solves either the full or the linearized Euler equations in a conservative and perturbed form (with a splitting of the complete variables into a 'frozen' mean flow and a 'fluctuating' perturbation). The solver employs high-order, finite-difference (FD) operators, as well as a third-order, multi-stage, Runge-Kutta time-marching scheme. The code deals with multiblock structured grids with one-to-one interfaces. Finally, the solver includes the usual boundary conditions (reflection by solid walls, non-reflecting and free-field radiation, ${ }^{10,22}$ etc.), as well some unique to specific applications (such as the Non Reflective Interface technique ${ }^{27}$ that was extensively used in the present effort, for properly forcing the CAA stage with the near-field datasets). The present effort benefits from a new class of finite difference (FD) schemes that were recently developed. ${ }^{28}$ The higher accuracy of these Intrinsically Optimized FD (IOFD) schemes produce accurate solutions on courser CAA grids than can be used with standard, order-property operators of the same stencil width.

\section{Subcategory 1: Scattering of Noise by a Sphere}

Subcategory 1: Scattering of Noise by a Sphere consists of a point quadrupole located near a sphere in a quiescent, homogeneous medium. The purpose of Subcategory 1 is primarily for use as a code verification exercise demonstrating the properties and order of the codes. For this subcategory, participants were provided a Fortran code that could calculate the acoustic solution on a box around the point quadrupole and sphere, including any scattering/reflection caused by the sphere. The solution on this box was then provided to the propagation algorithms which were required to calculate the noise on an arc outside the box. The noise prediction was then compared to the analytical solution. Participants were asked to provide information about the computational resources including architecture, details on processing unit(s), wall-clock time, and memory required. Figure 4 shows a schematic of Subcategory 1 including the point quadrupole, the sphere which acts as a scattering body, and the six-sided computational surface.

Subcategory 1 involved three participants: upacsAcoustics, TD-FAST, and sAbrinA. Figure 5 shows a snapshot of acoustic pressure predicted by upacsAcoustics on a sphere surrounding the point quadrupole. TD-FAST and sAbrinA provided similar data. Table 1 tabulates the different approaches to subcategory 1 , including the number of observers where a prediction was made, the architecture of the computers utilized, and the total amount of memory required to perform the prediction. upacsAcoustics and TD-FAST predicted the noise on a similar number of observers, but since sAbrinA is a CAA method, the solution at every cell used in the computation is available, thus the number of observers for sAbrinA is significantly higher. The TDFAST and sAbrinA codes required significantly more memory than upacsAcoustics and more computational effort. Table 2 summarizes the wall clock time and error of the computations. All three methods produced similar error; however, the wall clock time between the methods is very different. Since sAbrinA is a CAA method that must calculate the solution on a mesh encompassing the entire propagation region, there are significantly more observer positions. The wall clock time per observer per time step is the smallest. TDFAST requires the most computational resources per observer, but this metric is biased because of the number of observers associated with the CAA method. In most instances, the solution is not needed at all the points in the CAA mesh. 

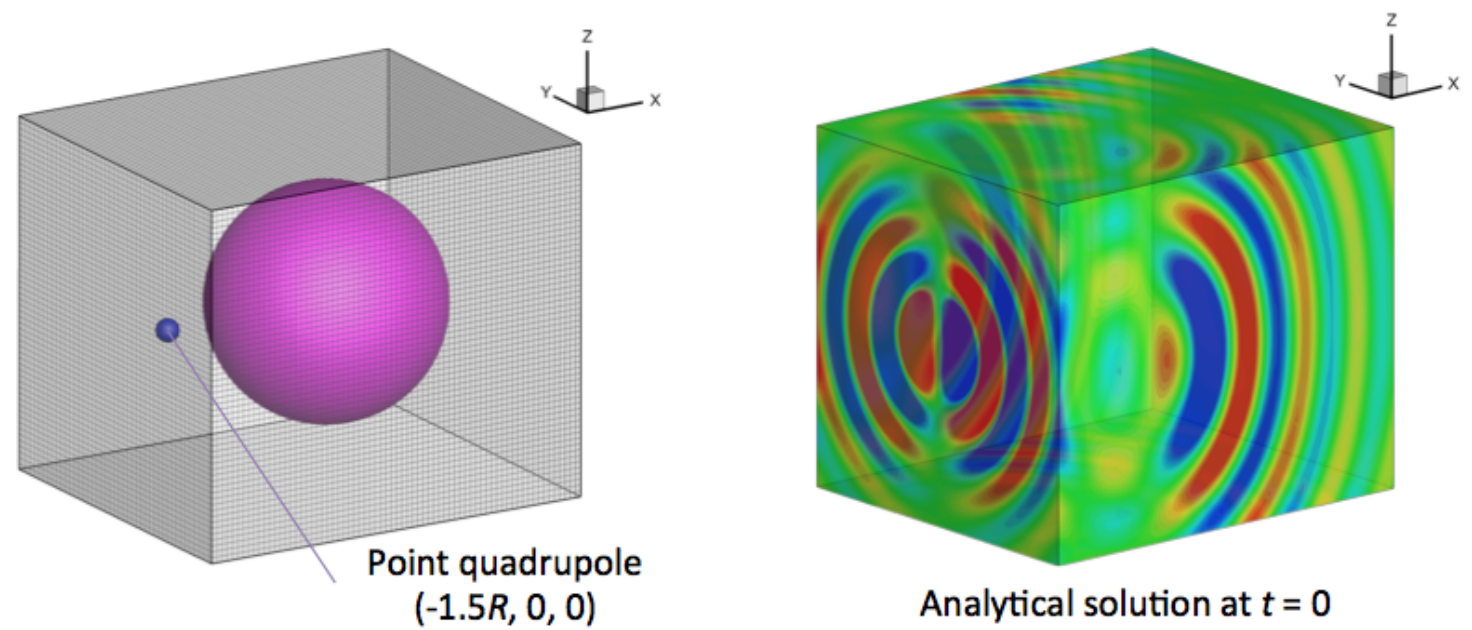

Figure 4. Schematic of Subcategory 1 which includes a point quadrupole near a sphere of radius $R$ and the computational surfaces surrounding both. Also shown is the acoustic solution on the computation surfaces at $t=0$.

\begin{tabular}{|l|c|c|c|c|c|}
\hline & Approach & \# Observers & Architecture & \# Cores & Memory (Mbytes) \\
\hline upacsAcoustics & IM & 342 & Not Available & 1 & 35 \\
\hline TD-FAST & BEM & 342 & Nvidia M2090 GPU & 24 & 800 \\
\hline sAbrinA & CAA & 520000 & Intel i7-3610 QM CPU & 1 & 9,182 \\
\hline
\end{tabular}

Table 1. Summary of participants' setups in subcategory 1.

Comparisons of efficiency and speed between implementations of methods that are significantly different in approach is difficult. Due to time limitations, variations in computational setup, such as time step size and mesh size were not included in this work. In the future, more exploration into the potential variation due to these parameters will be explored. Even so, the above represents a first step in this comparison. In future workshops, additional participation will result in a better understanding of the variation in computational requirements. Also, improvements will be made to Subcategory 1, including a more complex noise calculation (such as a scattering body outside the source region) and some metric for benchmarking the computational requirements of the predictions. These efforts would result in a more consistent and representative metric for all methods.

\section{Subcategory 2: Nose Landing Gear}

Subcategory 2 was created to quantify the variability in predicted spectra from propagation algorithms given a more realistic input. Participants were provided a CFD solution of the flow around a nose landing gear geometry (the Airbus-ONERA LAGOON configuration used in BANC-II Category 5). ${ }^{29-31}$ The solution, provided as an internet download of dimensional flow quantities, included on-body (impermeable) and off-body (permeable) surfaces, the latter of 


\begin{tabular}{|l|c|c|}
\hline & Wall Clock / Observer / Time Step $(\mu$ sec $)$ & Mean-RMS Error \\
\hline upacsAcoustics & 20.3 & $4.3369 e^{-6}$ \\
\hline TD-FAST & 1950 & $2.7160 e^{-6}$ \\
\hline sAbrinA & 8.6 & $1.782 e^{-7}$ \\
\hline
\end{tabular}

Table 2. Summary of wall clock time and mean-rms error for participants of subcategory 1.

which is designed as a nearly cubic interface encompassing the wheels and is composed of 6 panels shown in Fig 6. A Fortran 95 code that reads the datasets was also provided to the participants for ease in handling the data. The impermeable and permeable datasets contained 20100 and 14390 time steps, respectively, both with a common time step spacing of 5e-6 seconds. Participants were permitted to downsample the CFD solution in time and/or space as needed to help characterize the dependence of their predictions on spatial and/or temporal resolution. Participants were asked to provide the acoustic pressure at 5 far-field observer locations (designated F1 through F5), 4 near-field observer locations (designed N1 through N4), and at an observer at the midpoint (origin) between the wheels inside the surfaces (designated as the origin). Supplied results were to include pressure time signals and power spectral densities. The observer locations, with the exception of the origin, coincided with measurement locations used in ONERA's open-jet anechoic windtunnel CEPRA19 in Saclay, France. ${ }^{30}$

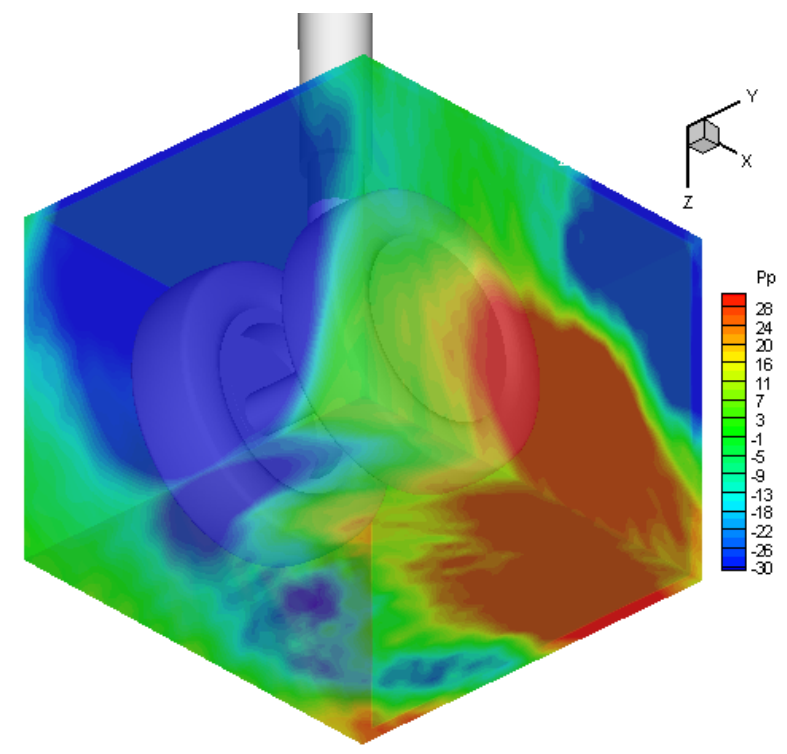

Figure 6. Permeable interfaces extracted from CFD solution of flow around LAGooN geometry.
Subcategory 2 included three participants: two IM solvers (ANOPP2-F1A and upacsAcoustics) and one CAA solver (sAbrina). ANOPP2-F1A and upacsAcoustics utilized the entire CFD solution (not downsampling in space and time); however, sAbrina utilized a solution that was downsampled in space (1:2 ratio) and time (sufficient to resolve a maximum frequency of $3 \mathrm{kHz}$ ). Figure 7 shows a snapshot of predicted acoustic pressure time histories computed using the permeable surface data. Although there are significant variations in the three predictions, they show similar trends. Some of the variation can be attributed to the downsampled solution provided to the sAbrina code. It is also interesting to note that even though upacsAcoustics and ANOPP2-F1A utilize the exact same formulation for noise propagation, there are differences in the predictions that are non-trivial. All three methods included different observer time ranges and averaging techniques that complicated comparisons of the supplied spectra. Therefore, the spectra were computed in a consistent fashion based on the supplied time history data. A common temporal sample of 0.069 seconds for predictions based on the impermeable surfaces and 0.042 seconds for predictions based on the permeable surfaces was chosen. This resulted in 1666 samples from upacsAcoustics and sAbrinA and 3400 from ANOPP2-F1A. A Hanning window was applied to all time histories before calculating a power spectral density. Figures 8 and 9 show the power spectral densities from the permeable surface calculations using the full data record. The results are presented in a narrow $23.8 \mathrm{~Hz}$ bin width. It is difficult to assess the three results using the narrow bin width due to the high variability. A longer time history providing sufficient averaging, would result in a smoother power spectral density curve; however, due to the limited duration of source time history in the CFD input, a longer predicted time range is not possible. Therefore, an FFT averaging scheme was employed where the time histories were separated into 5 FFT averaging blocks for predictions based on the permeable dataset and 8 FFT averaging blocks for predictions based on the impermeable dataset. Both averaging techniques employed $0 \%$ overlap resulting in a $120 \mathrm{~Hz}$ bin width.

Figure 10 shows predictions at F1 through F5 from the three participants using a permeable surface solu- 


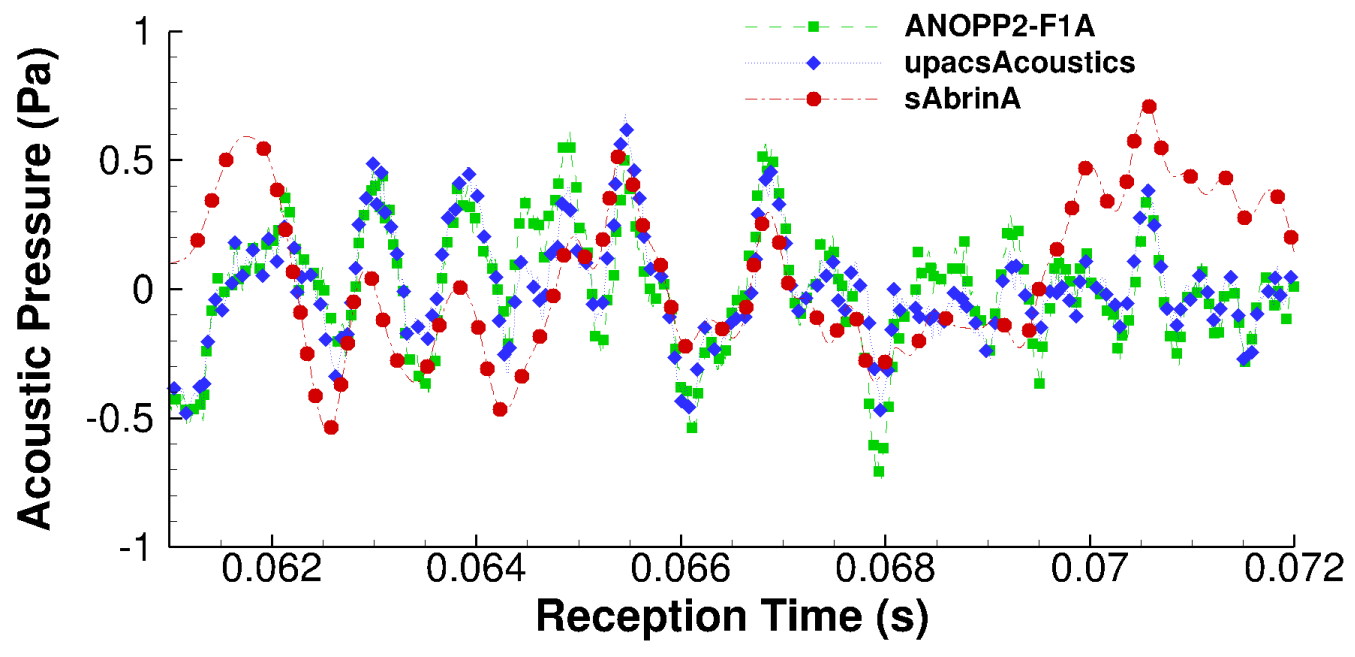

Figure 7. Snapshot of acoustic pressure time history predictions at F1 from ANOPP2-F1A, upacsAcoustics, and sAbrinA codes using permeable surfaces in shape of a box around landing gear.
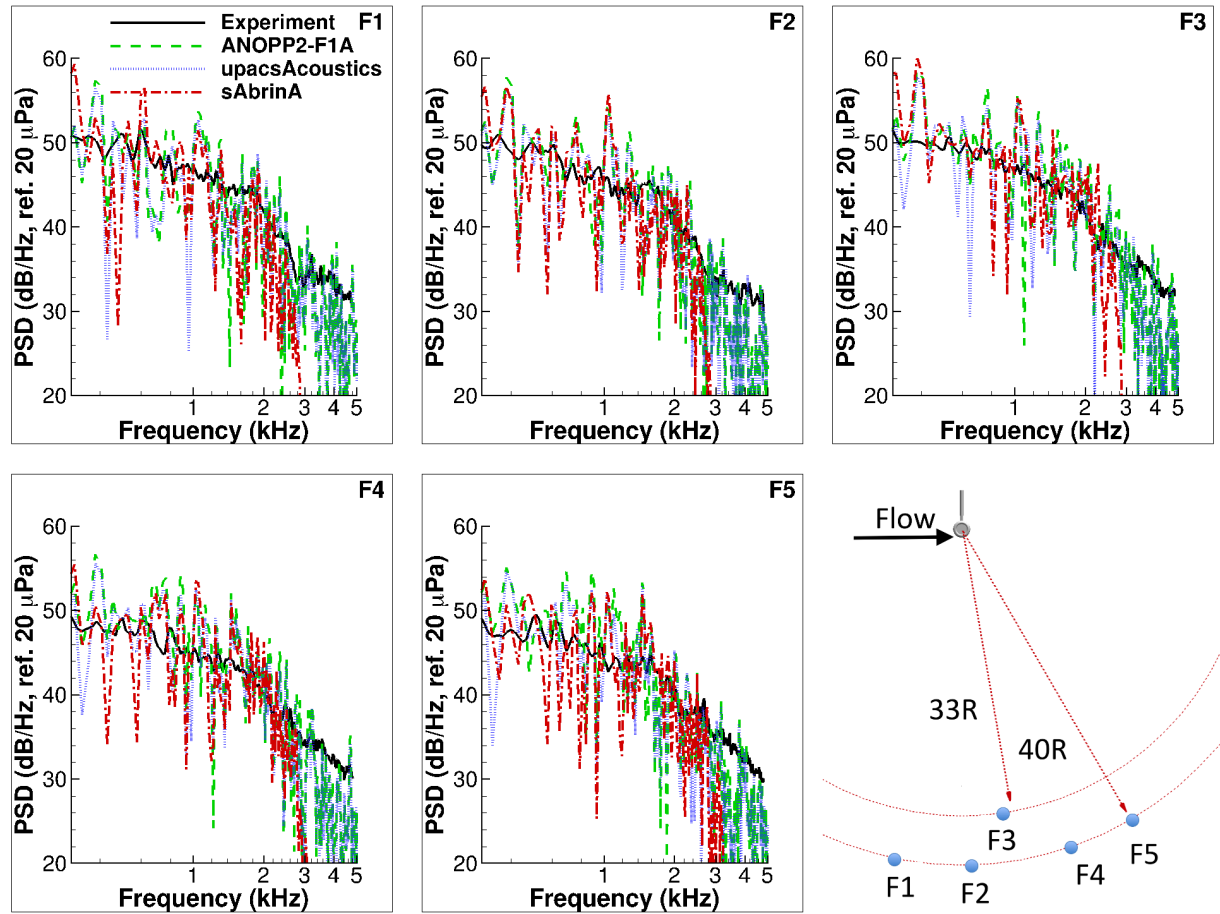

Figure 8. Power spectral densities from ANOPP2-F1A, upacsAcoustics, and sAbrinA codes using permeable surfaces at microphone locations F1 through F5. No FFT averaging was applied. 

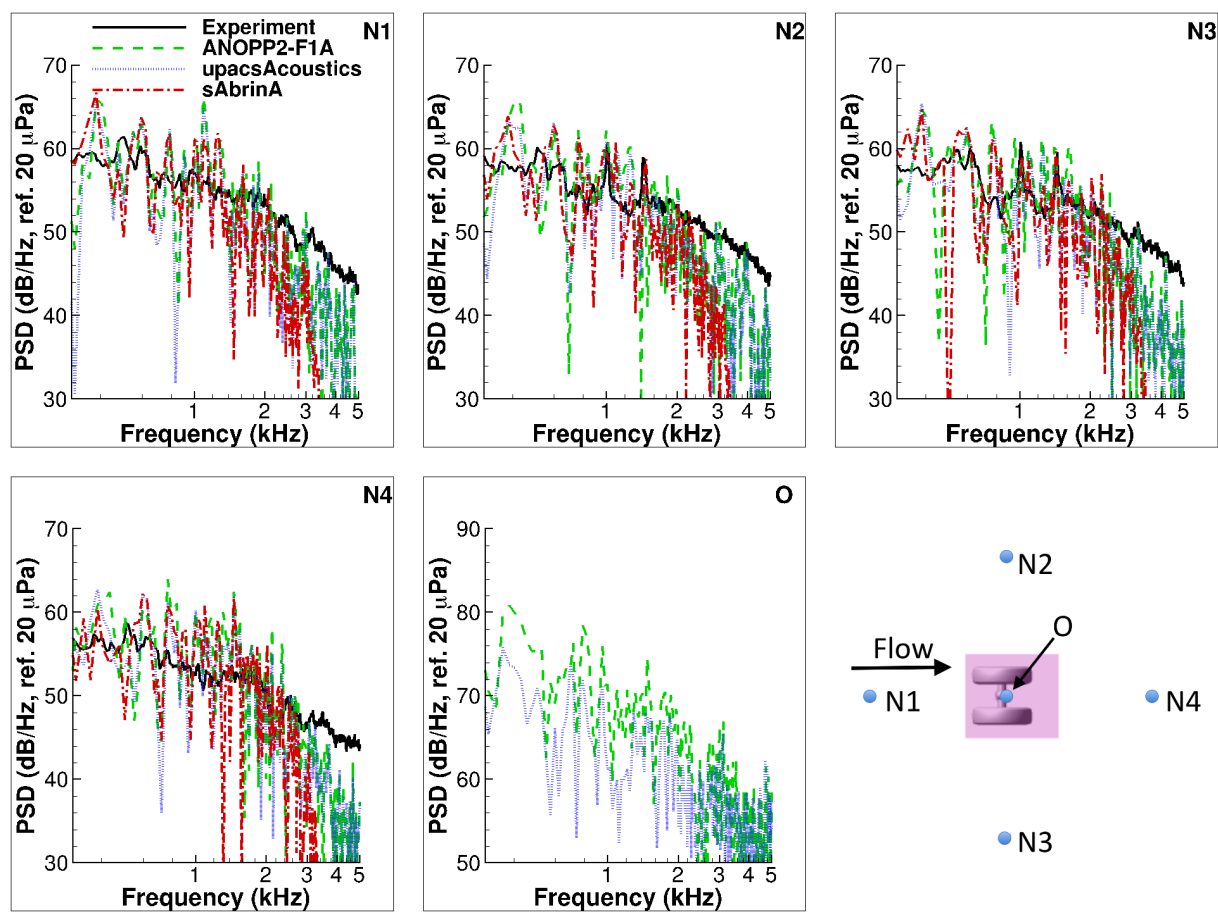

Figure 9. Power spectral densities from ANOPP2-F1A, upacsAcoustics, and sAbrinA codes using permeable surfaces at microphone locations N1 through N5 and the origin. No FFT averaging was applied.

tion as input. For all observer positions, all predictions agree very well with the experiment and each other. It is interesting to note that all predictions tend to overpredict around and below $1 \mathrm{kHz}$ and underpredict in the high frequencies. Since all methods are behaving similarly, this trend indicates a dependence on the simulation data used as input rather than some characteristic of the codes. Undoubtedly, the diffusive nature of the CFD simulation results in an artificial decay of high-frequency waves (above $2 \mathrm{kHz}$ ). The sAbrinA results decay even faster at high frequencies, most likely because of subsampling the input data, but possibly because of diffusion in the calculation itself. Figure 11 shows the predictions compared to measurements at the four near-field microphone locations, N1 through N4. The agreement with experiment for all three solvers is very good, particularly at the upstream position N1. For the near-field microphones N1, N2, and $\mathrm{N} 3$, all predictions agree very well with the experiment in the low- and mid-frequencies. At the downstream location, N4, all codes significantly overpredict the lower frequencies. Figure 11 also shows the prediction at the origin from the two IM solvers, ANOPP2-F1A and upacsAcoustics. Predictions using the FW-H equation theoretically should predict exactly zero inside the surface and both codes do not, with ANOPP2F1A predicting significantly higher levels than upacsAcoustics. Possible causes for the discrepancy include: neglecting the viscous terms in the Lighthill stress tensor, neglecting the quadrupole term, and numerical error. The exact cause is unknown, but will be a focus of future efforts.

Figures 12 and 13 show the far-field and near-field predictions from ANOPP2-F1A and upacsAcoustics using the impermeable dataset. For all microphones the high frequencies are underpredicted by all the codes, especially at the near-field microphones. For all microphones, far-field and near-field, the low- and mid-frequency levels are predicted extremely well by both codes (the predictions by ANOPP2-F1A are hidden by the predictions by upacsAcoustics), including the N4 position where the predictions using the permeable dataset overpredict the levels compared to the experiment. This suggests that the overprediction in the downstream location and in the far-field microphones at the low frequencies using the permeable datasets is due to the conditioning of the rear permeable surface and that using permeable datasets in the future requires a correction in order to accurately predict in the low frequency. Figure 13 also shows prediction from both codes at the origin, which is placed at the center of the hub. Both ANOPP2-F1A and upacsAcoustics predict significantly high values due to the closeness of the surface when theoretically they 

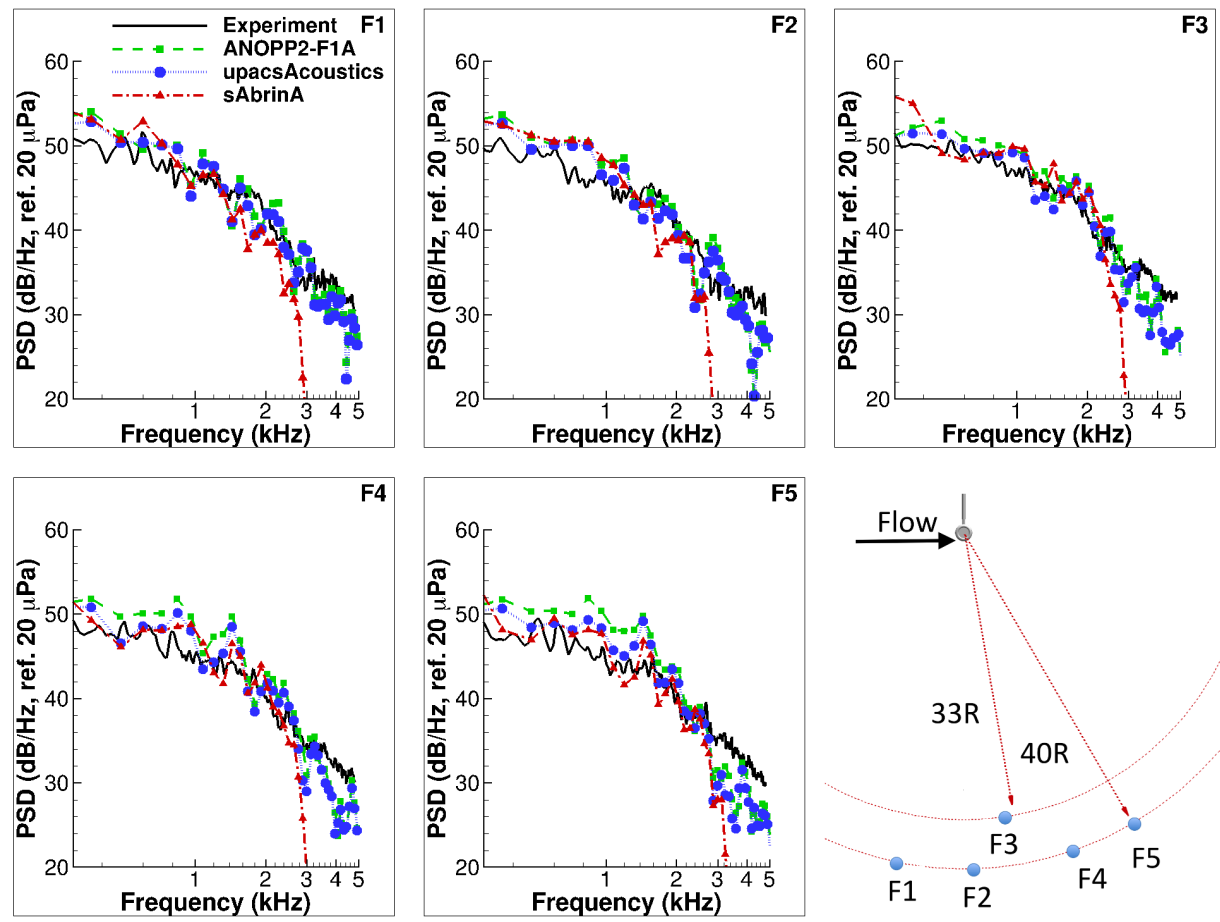

Figure 10. Power spectral densities at microphone locations F1 through F5 from ANOPP2-F1A, upacsAcoustics, and sAbrinA codes using permeable surfaces. Five FFT averages were applied resulting in a $120 \mathrm{~Hz}$ bin width.
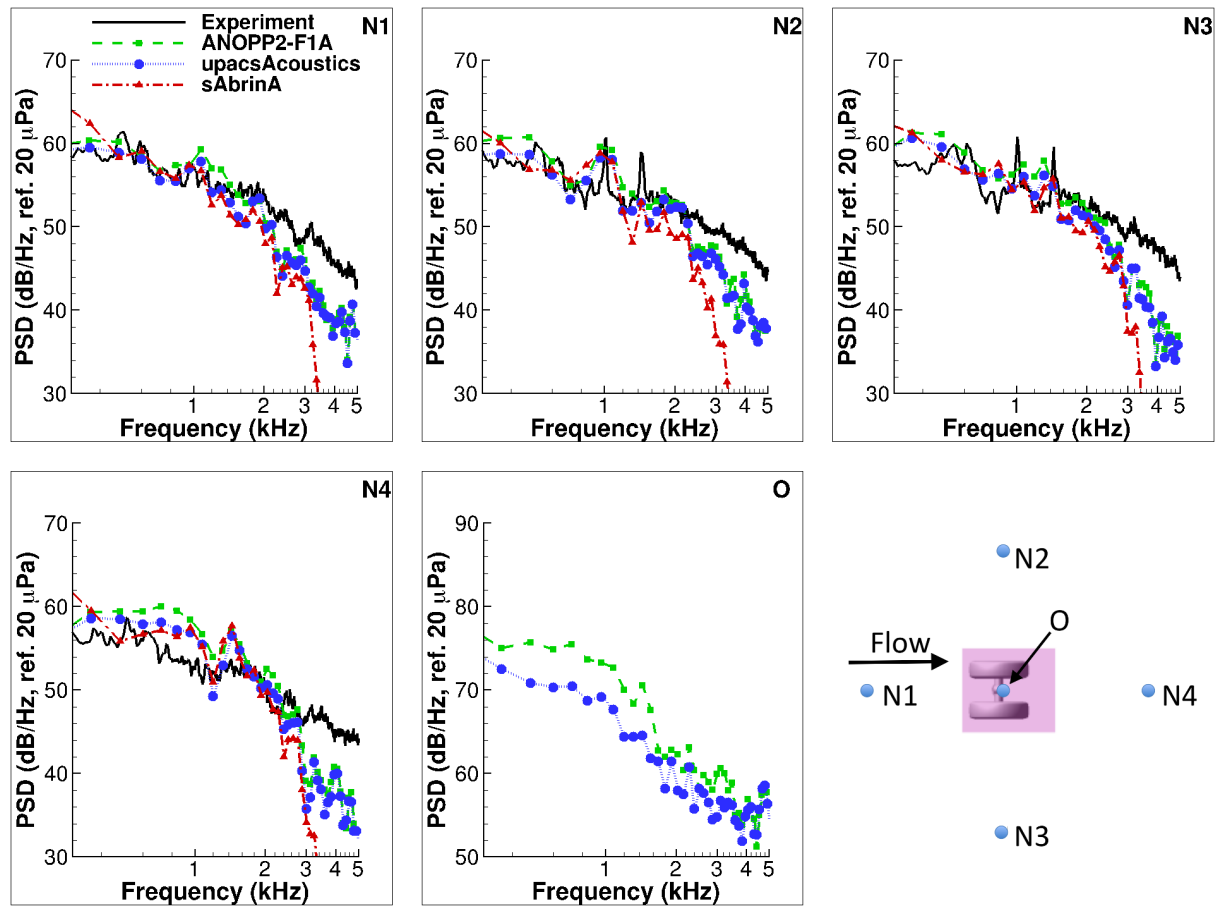

Figure 11. Power spectral densities at microphone locations N1 through N5 and the origin from ANOPP2F1A, upacsAcoustics, and sAbrinA codes using permeable surfaces. Five FFT averages were applied resulting in a $120 \mathrm{~Hz}$ bin width. 
should be predicting exactly zero.
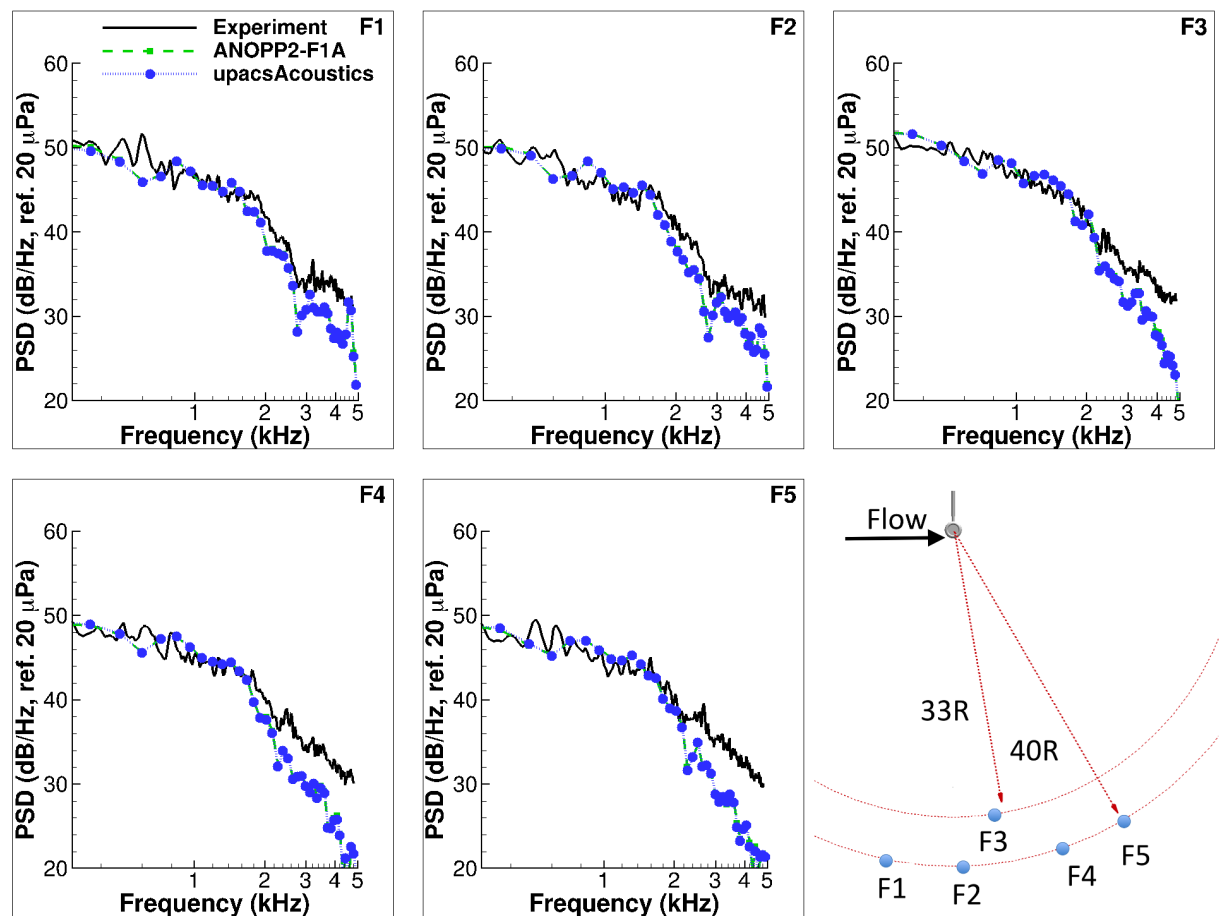

Figure 12. Power spectral densities at microphone locations F1 through F5 from ANOPP2-F1A and upacsAcoustics codes using impermeable surfaces. Nine FFT averages were applied resulting in a $120 \mathrm{~Hz}$ bin width.

An objective of Category 8 of the BANC-III workshop is to assess the solution variability caused by the propagation phase of CFD-based noise prediction. As such, a first step in assessing the variability is to compare the output from propagation codes provided the exact same input. Figures 14 and 15 show the difference between ANOPP2-F1A and upacsAcoustics predictions at the far-field and near-field microphones (using the impermeable surface as input) expressed as a $\triangle$ SPL about an average SPL of the two predictions; thus, the symmetric nature of the curves. Due to the high variance at extreme low and high frequencies, only frequencies between $0.24 \mathrm{kHz}$ and $3 \mathrm{kHz}$ were analyzed. Across the available frequencies and all observer positions, the variability of the predictions is less than $0.25 \mathrm{~dB}$ suggesting that there is good agreement between the two solvers when using impermeable data. Figures 16 and 17 show the far-field and near-field variability between ANOPP2-F1A, upacsAcoustics, and sAbrinA using the permeable data as input. Across all frequencies and observer positions, the variability is significant, particularly as the frequency increases. This variation in the high frequencies is partially due to the downsampled solution provided to sAbrina and suggests that the current analysis may be problematic because of the particular way the different propagation approaches were applied to this problem.

While spectral deviation at all the observer positions as a function of frequency is interesting and allows an identification of key problem areas, it is advantageous to reduce this deviation to a single number, thus quantifying the variability in the propagation phase of noise prediction. The authors admit that the participation to Subcategory 2 is sparse and an estimation of the overall variability would benefit from more participation. Regardless, an overall estimate of the variability is calculated. Equation 11 shows the process for calculating the standard deviation across all microphones and frequencies (results from permeable dataset only). Taking the standard deviation for near- and far-field predictions results in a standard deviation of 1.5 $\mathrm{dB}$ and $2.7 \mathrm{~dB}$ respectively. Due to the nature of the study, the authors feel that rounding up to $2 \mathrm{~dB}$ and $3 \mathrm{~dB}$ for the near-field and far-field variability is appropriate at this time. This variability can be directly 

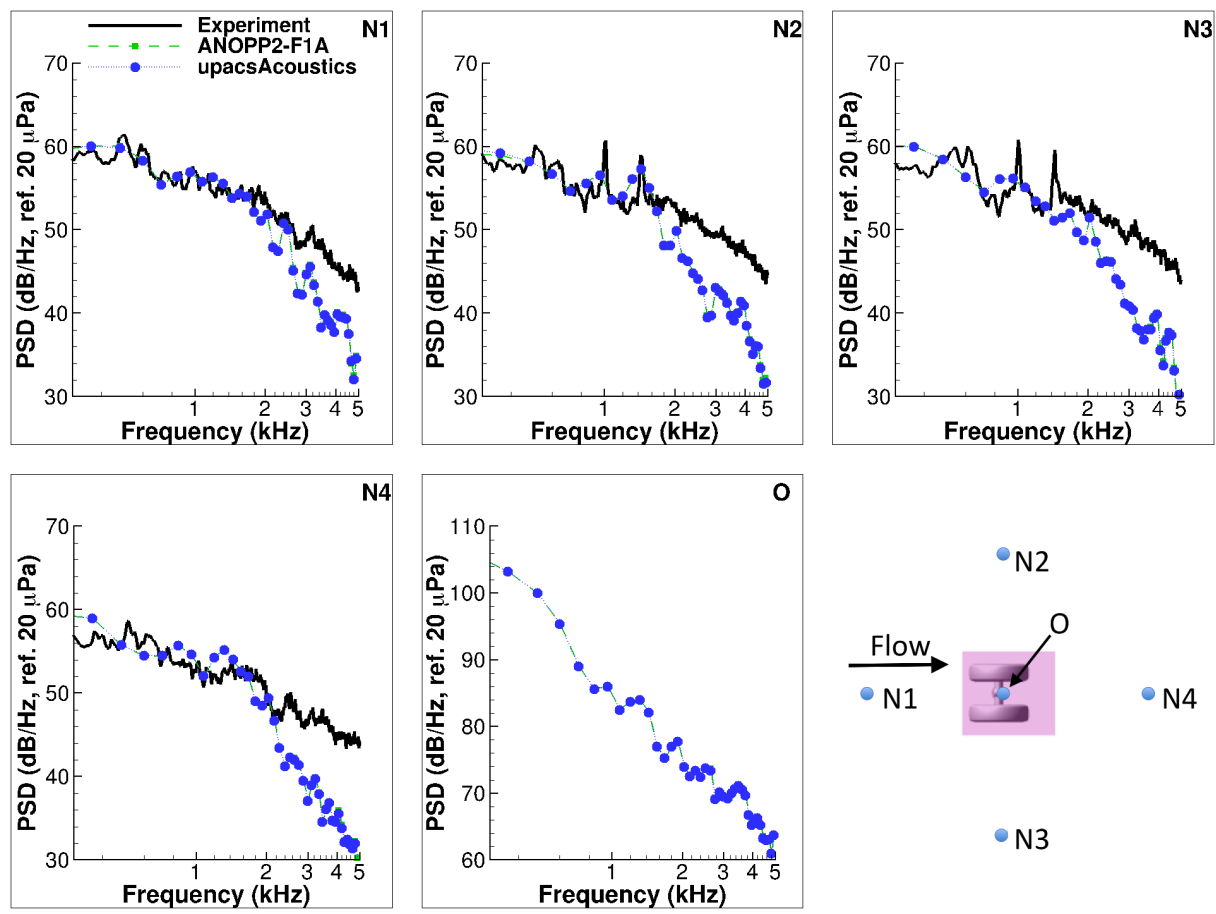

Figure 13. Power spectral densities at microphone locations N1 through N5 and the origin from ANOPP2-F1A and upacsAcoustics codes using impermeable surfaces. Nine FFT averages were applied resulting in a $120 \mathrm{~Hz}$ bin width.
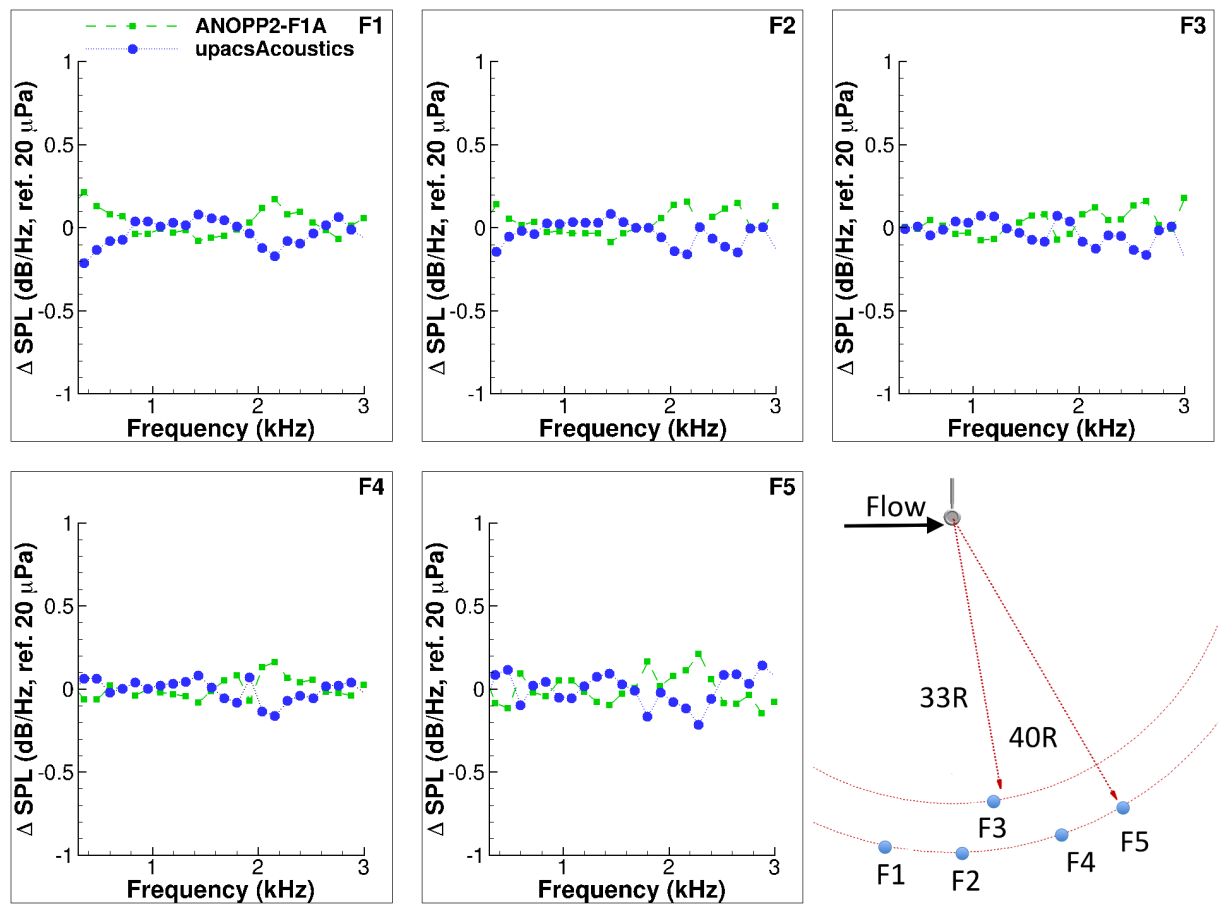

Figure 14. $\triangle$ SPL between ANOPP2-F1A and upacsAcoustics predictions at the far-field microphones using the impermeable surfaces as input. 

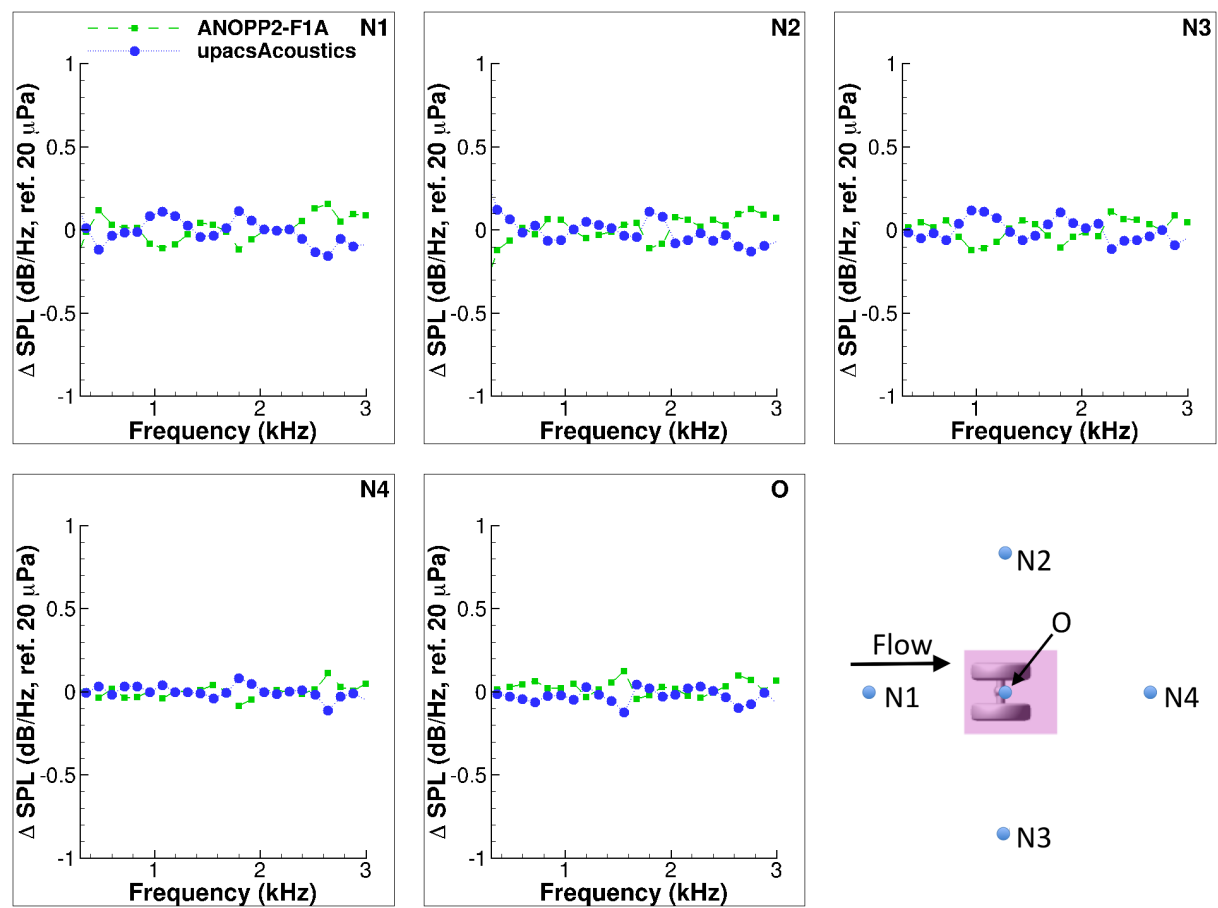

Figure 15. $\triangle$ SPL between ANOPP2-F1A and upacsAcoustics predictions at the near-field microphones using the impermeable surfaces as input.
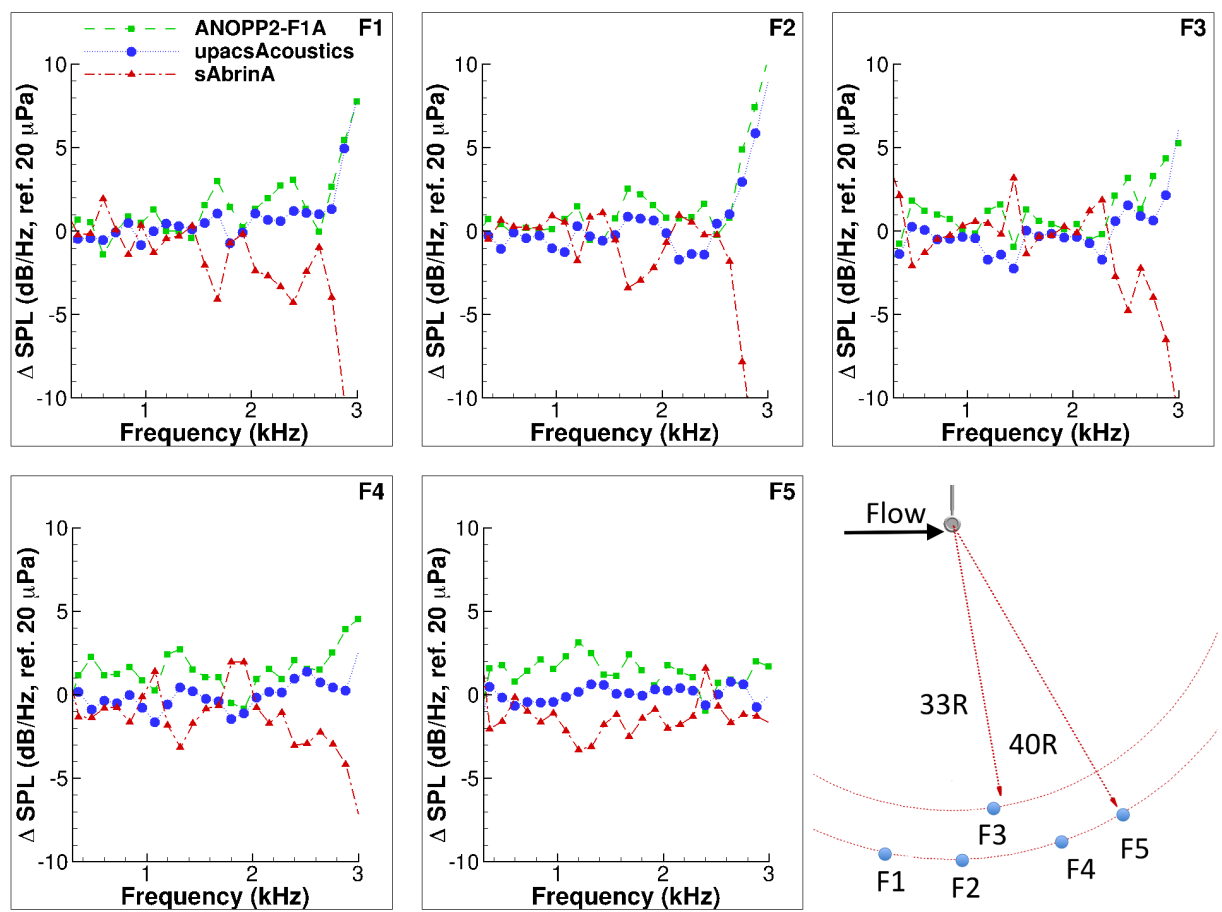

Figure 16. $\triangle$ SPL between ANOPP2-F1A, upacsAcoustics, and sAbrinA predictions at the far-field microphones using the permeable surfaces as input. 

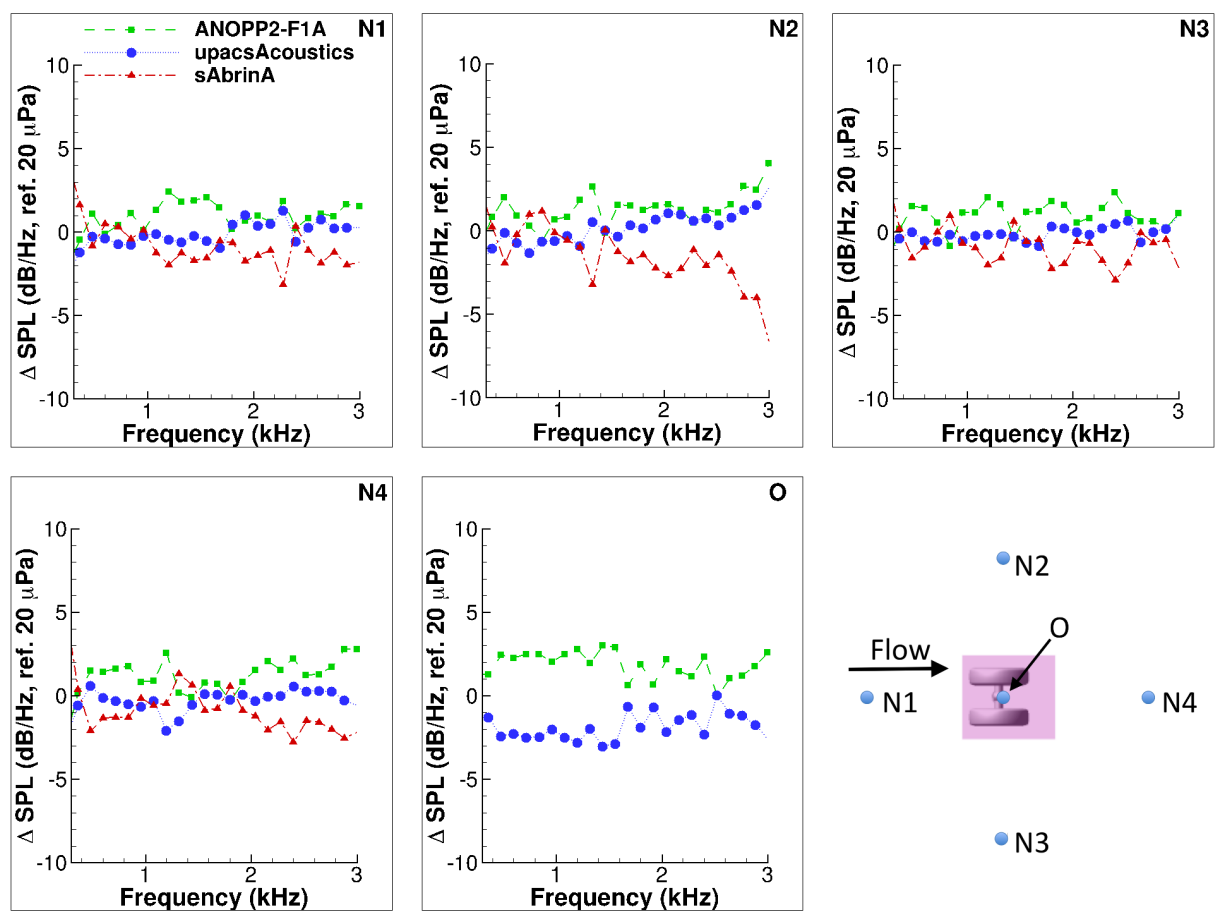

Figure 17. $\triangle$ SPL between ANOPP2-F1A, upacsAcoustics, and sAbrinA predictions at the near-field microphones using the permeable surfaces as input.

attributed to the choice of propagation algorithm when performing a CFD-based noise prediction.

$$
\sigma=\sqrt{\frac{\sum_{i=1}^{N_{o b s}} \sum_{j=1}^{N_{\text {bins }}}\left(\Delta S P L_{i j}-\mu_{i}\right)^{2}}{N_{\text {obs }} \times N_{\text {bins }}}} \quad \text { where } \quad \mu_{i}=\frac{\sum_{k=1}^{N_{\text {bins }}} \Delta S P L_{i k}}{N_{\text {bins }}}
$$

\section{Conclusion and Future Work}

This paper presents results from the BANC-III workshop, which explores variability in the propagation phase of CFD-based noise prediction. This included two test cases: an analytical solution of a quadrupole source near a sphere and a computational solution around a nose landing gear. Excellent agreement was found for the analytical test case between Integral Method solvers using the impermeable surface data. For the computational solution around a nose landing gear, differences of 2-3dB were common for impermeable surfaces. Potential causes of variability include error in the CFD solution provided as input, how the approaches used the CFD data, implementation details, and spurious signals in the IM solvers. Obviously, additional participation in future BANC workshops and additional categories will improve the assessment of the variability.

This represents a first step in quantifying the importance of the propagation phase of CFD-based noise prediction. In the future, additional categories will be added, in particular ones that focus on calculating the scattering/shielding of noise and accounting for the influence of flow heterogeneities in the mid-field region. In addition to new categories, future work will involve modifying the resolution of the input data and adding more complex configurations of both Subcategory 1 and 2 that will allow a focused assessment of the variability due to influences such as computational reliability and flow characteristics. This will allow for improved understanding and assessment of the validity of predictions, including frequency ranges and observer positions. 


\section{Acknowledgements}

The authors would like to acknowledge Dr. Meelan Choudhari for his guidance and support during the BANC workshops and Dr. Fang Hu for his participation in Subcategory 1. Regarding Subcategory 2, the authors acknowledge Dr. Saloua Ben Khelil (ONERA) for having granted them access to the unsteady CFD data coming from the LAGooN project, which was supported and led by Airbus. Similarly, Dr. Bastien Caruelle (Airbus) and Dr. Eric Manoha (ONERA) are acknowledged for their help regarding the dissemination and use of LAGooN experimental data. Authors would like to acknowledge Dr. Kazuomi Yamamoto (JAXA) for his support to this category. Additionally, Dr. Kazuhisa Amemiya (JAXA) is acknowledged for performing all the numerical processing done by the JAXA team. The NASA team was supported by the Transformative Tools and Technology Project in the Transformative Aeronautics Concepts Program.

\section{References}

\footnotetext{
${ }^{1}$ Farassat, F. and Myers, M. K., "Extension of Kirchhoff's Formula to Radiation from Moving Surfaces," Journal of Sound and Vibration, Vol. 123, No. 3, 1988, pp. 451-461.

${ }^{2}$ Farassat, F. and Myers, M. K., "The Kirchhoff Formula for Supersonically Moving Surface," AIAA Paper 1995-062, $1^{\text {st }}$ Joint CEAS/AIAA Aeroacoustic Conference (16 ${ }^{\text {th }}$ AIAA Aeroacoustics Conference), 1995.

${ }^{3}$ Lighthill, M. J., "On Sound Generated Aerodynamically, I: General Theory," Proceedings of the Royal Society. A, Mathematical, Physical, and Engineering Sciences, Vol. 211, 1952, pp. 564-587.

${ }^{4}$ Ffowcs Williams, J. E. and Hawkings, D. L., "Sound Generated by Turbulence and Surfaces in Arbitrary Motion," Philosophical Transactions of the Royal Society, Vol. 264, No. 1151, 1969, pp. 321-342.

${ }^{5}$ Agarwal, A., Dowling, A. P., Shin, H.-C., and Graham, W., "Ray-Tracing Approach to Calculate Acoustic Shielding by a Flying Wing Airframe," AIAA Journal, Vol. 45, No. 5, 2007, pp. 1080-1090.

${ }^{6}$ Chen, H., Crutchfield, W. Y., Gimbutas, Z., Greengard, L. F., Ethridge, J. F., Huang, J., Rokhlun, V., Yarvin, N., and Zhao, J., "A wideband fast multipole method for Helmholtz equation in three dimensions," Journal of Computational Physics, Vol. 216, 2006, pp. 300-325.

${ }^{7} \mathrm{Hu}$, F. Q., "Further Development of a Time Domain Boundary Integral Equation Method for Aeroacoustic Scattering Computation," 20 ${ }^{\text {th }}$ AIAA/CEAS Aeroacoustics Conference, AIAA 2014-3194, June 16-20 2014.

${ }^{8}$ Tinetti, A. F., Dunn, M. H., and Pope, D. S., Fast Scattering Code (FSC) User's Manual, Version 2.0, October 2006, NASA CR 2006-214510.

${ }^{9}$ Lee, S., Brentner, K. S., and Morris, P. J., "Acoustic Scattering in the Time Domain Using an Equivalent Source Method," AIAA Journal, Vol. 48, No. 12, December 2010, pp. 2772-2780.

${ }^{10}$ Redonnet, S., Manoha, E., and Sagaut, P., "Numerical Simulation of Propagation of Small Perturbations Interacting with Flows and Solid Bodies," AIAA-2001-2223, $7^{\text {th }}$ AIAA/CEAS Aeroacoustics Conference (22nd AIAA Aeroacoustics Conference), May 28-30 2001.

${ }^{11}$ Choudhari, M., "Third AIAA Workshop on Benchmark Problems for Airframe Noise Computations (BANC - III)," https://info.aiaa.org/tac/ASG/FDTC/DG/BECAN_files_/BANCIII.htm, 2013.

${ }^{12}$ Farassat, F., "Introduction to Generalized Functions With Applications in Aerodynamics and Aeroacoustics," Technical Paper 3428, National Aeronautics and Space Administration, Langley Research Center, Hampton, VA 23681-0001, May 1994.

${ }^{13}$ Imamura, T., Hirai, T., Amemiya, K., Yokokawa, Y., Enomoto, S., and Yamamoto, K., "Aerodynamic and aeroacoustic simulations of a two-wheel landing gear." Procedia Engineering, Vol. 6, 2010, pp. 293-302.

${ }^{14}$ Lopes, L. V. and Burley, C. L., "Design of the Next Generation Aircraft NOise Prediction Program: ANOPP2," AIAA2011-2854, $17^{\text {th }}$ AIAA/CEAS Aeroacoustics Conference (32 ${ }^{\text {nd }}$ AIAA Aeroacoustics Conference), June 5-8 2011.

${ }^{15} \mathrm{Hu}$, F. Q., "An Efficient Solution of Time Domain Boundary Integral Equations for Acoustic Scattering and its Acceleration by Graphics Processing Units," AIAA-2013-2018, $19^{\text {th }}$ AIAA/CEAS Aeroacoustics Conference (34 ${ }^{\text {th }}$ AIAA Aeroacoustics Conference), May 27-29 2013.

${ }^{16}$ Gray, J. S., Moore, K. T., and Naylor, B. A., "OPENMDAO: An Open Source Framework for Multidisciplinary Analysis and Optimization," 3th AIAA/ISSMO Multidisciplinary Analysis and Optimization Conference, AIAA-2010-9101, August 2010.

${ }^{17}$ Hennes, C. C. and Brentner, K. S., "The Effect of Blade Deformation on Rotorcraft Acoustics," Presented at the $31^{\text {st }}$ European Rotorcraft Forum, September 13-15 2008.

${ }^{18}$ Farassat, F., "Derivation of Formulations 1 and 1A of Farassat," NASA TM 2007-214853, NASA Langley, 2007.

${ }^{19}$ Farassat, F. and Casper, J., "Broadband noise prediction when turbulence simulation is available-Derivation of Formulation 2B and its statistical analysis," Journal of Sound and Vibration, Vol. 331, 2012, pp. 2203-2208.

${ }^{20}$ Lee, S., Brentner, K. S., and Farassat, F., "Analytic Formulation and Numerical Implementation of an Acoustic Pressure Gradient Prediction," Journal of Sound and Vibration, Vol. 319, No. 3-5, 2009.

${ }^{21}$ Ghorbaniasl, G., Carley, M., and Lacor, C., "Acoustic Velocity Formulation for Sources in Arbitrary Motion," AIAA Journal, Vol. 51, No. 3, 2013, pp. 632-642.

${ }^{22}$ Redonnet, S., Simlation de la propagation acoustique en présence d'écoulements quelconques et de structures solides, par résolution numérique des équations d'Euler, Ph.D. thesis, Université Bordeaux I, December 2001.

${ }^{23}$ Redonnet, S., Desquesnes, G., Manoha, E., and Parzani, C., "Numerical Study of Acoustic Installation Effects with a CAA Method," AIAA Journal, Vol. 48, No. 5, May 2010, pp. 929-937.
} 
${ }^{24}$ Reboul, G. and Polacsek, C., "Towards Numerical Simulation of Fan Broadband Noise Aft Radiation from Aeroengines," AIAA Journal, Vol. 48, No. 9, September 2010, pp. 2038-2048.

${ }^{25}$ Redonnet, S. and Druon, Y., "Computation AeroAcoustics of Realistic Co-Axial Engines," AIAA Journal, Vol. 50, No. 5, May 2012, pp. 1029-1046.

${ }^{26}$ Clair, V., Polacsek, C., Garrec, T. L., Reboul, G., Gruber, M., and Joseph, P., "Experimental and Numerical Investigation of Turbulence-Airfoil Reduction Using Wavy Edges," AIAA Journal, Vol. 51, No. 11, November 2013, pp. $2695-2713$.

${ }^{27}$ Redonnet, S., Lockard, D. P., Khorrami, M. R., and Choudhari, M. M., "The Non-Reflective Interface: An Innovative Forcing Technique for Computational Acoustic Hybrid Methods," Submitted for publication in International Journal for Numerical Methods in Fluids, 2015.

${ }^{28}$ Cunha, G. and Redonnet, S., "A Novel Optimization Technique for Explicit Finite-Difference Schemes with Application to AeroAcoustics," Accepted for publication in International Journal for Numerical Methods in Fluids, 2015.

${ }^{29}$ Manoha, E., Bulté, J., and Caruelle, B., "LAGOON: an Experimental Database for the Validation of CFD/CAA Methods for Landing Gear Noise Prediction," AIAA-2008-2816, $14^{\text {th }}$ AIAA/CEAS Aeroacoustics Conference (29 ${ }^{\text {th }}$ AIAA Aeroacoustics Conference), May 5-7 2008.

${ }^{30}$ Manoha, E., Bulté, J., Ciobaca, V., and Caruelle, B., "LAGOON: Further Analysis of Aerodynamic Experiments and Early Aeroacoustics Results," AIAA-2009-3277, $17^{\text {th }}$ AIAA/CEAS Aeroacoustics Conference (32 ${ }^{\text {nd }}$ AIAA Aeroacoustics Conference), June $5-82009$.

${ }^{31}$ Deck, S., "Recent improvements in the Zonal Detached Eddy Simulation (ZDES) formulation," Theoretical and Computational Fluid Dynamics, Vol. 26, No. 6, 2012, pp. 523-550. 